\title{
Immunofluorescence Study of Germinal Centers in Mouse Lymph Nodes during the Secondary Immune Response*
}

\author{
Yoshifumi Ishit, Michio Mori and Teruo Fujimoto \\ Department of Pathology, $\dagger$ Sapporo Medical College, Sapporo
}

\begin{abstract}
IsHIr, Y., MoRI, M. and FuJimoto, T. Immunofluorescence Study of Germinal Centers in Mouse Lymph Nodes during the Secondary Immune Response. Tohoku J. exp. Med., 1970, 102(2), 143-157 - An immunofluorescence study carried out on the relationship between the mode of localization of antigen and that of specific antibody and $\gamma$-globulin in the germinal centers of mouse popliteal lymph nodes during the secondary immune response. The germinal centers of popliteal lymph nodes from mice sensitized secondarily with antigen (human $\gamma$-globulin) contained both antigen and $\gamma$-globulin in a similar distribution, giving lacy or reticular framework of immunofluorescene. Specific fixation of heterologous (guinea pig) complement in the germinal centers was also observed in a distribution similar to that of antigen and $\gamma$-globulin. These results strongly suggest the presence of antigenantibody complex mechanism in the germinal centers. The functional significance of this mechanism in the immune reaction is discussed. Large parenchymal cells of the germinal centers were devoid of specific staining for specific antibody as well as for $\gamma$-globulin, which may support the view that the germinal centers are not a source of plasma cells but a site of lymphocyte formation ___ germinal center; immunofluorescence; $\gamma$-globulin
\end{abstract}

It has been demonstrated that the formation and development of lymphatic tissue germinal centers are invariably produced by the optimal antigenic stimulation ${ }^{1-4}$ and that the antigen is specifically localized in germinal centers in close association with the reticular network composed of the cytoplasmic processes of specialized reticular cells. ${ }^{5-9}$ The antigen deposition in germinal centers has been considered to have causal relationship to the formation and development of germinal centers, ${ }^{6}$ although the detail of the relationship is not well known.

On the other hand, the presence of immunoglobulins in germinal centers has been reported by many authors, ${ }^{10-16}$ but an agreement is not reached as to the role of germinal centers in immunoglobulin synthesis. Some authors mentioned the production of immunoglobulins by cells of germinal centers, ${ }^{11,15}$ whereas others presented the evidence that immunoglobulins are closely associated with

Received for publication, June 27, 1970.

* Studies on the germinal center, II. Preliminary report of this work was presented at the IX Annual Meeting of Japanese Society of the Reticuloendothelial System in Hiroshima, June 3, 1969.

$\dagger$ Director: Prof. T. Fujimoto. 
the reticular network of germinal centers, with a distribution similar to that of antigen. ${ }^{12-14,16}$ An investigation of the role of germinal centers in immunoglobulin synthesis may contribute to elucidating their function in plasmocytopoiesis or lymphocytopoiesis.

In a previous ultrastructural study of germinal centers, we reported that the germinal center might not be the source of plasma cells but the site of systemic growth of lymphocytes. ${ }^{17}$ The purpose of this study is to evaluate the conclusion of the preceding paper from immunohistochemical aspect and further to investigate systematically the relationship between the mode of localization of antigen and that of specific antibody and immunoglobulin in the germinal centers of lymph nodes during the secondary immune response.

\section{Materials and Methods}

Animals. Forty-two mice of dd-strain of 3-4 months of age were used. They were maintained in a constant environment and allowed free access to regular chow and water ad libitum.

Antigen. Human $\gamma$-globulin (HGG) was purchased from Nutritional Biochemicals Corporation (Cleveland, Ohio).

Mouse $\gamma$-globulin (MGG). $\gamma$-Globulin fraction of the sera collected from eighty normal dd-mice was obtained by repeated ammonium sulfate fractionation followed by DEAE-cellulose column chromatography according to the method of Sober and Peterson. ${ }^{18}$

Guinea pig C'1. Guinea pig C'l was prepared and purified from normal guinea pig sera according to the method of Nelson et al..$^{19}$

Serology. The sera collected from each experimental mouse were titrated for antiHGG antibody by passive hemagglutination according to Daniel et al. ${ }^{20}$

Antisera. Specific antisera were prepared in albino rabbits against HGG, MGG and guinea pig C'l. The rabbits first received intradermal injection in their back with an emulsion of $20 \mathrm{mg}$ protein in complete Freund's adjuvant (Difco). They were boosted five weeks later in the same manner as in the first immunization. From 7 to 15 days after the secondary immunization a small amount of the sera was collected to determine the precipitin titers. When the amount of antibodies was satisfactory, the rabbits were exsanguinated by the heart puncture and the globulin fraction of each antiserum was precipitated with ammonium sulfate ( $35 \%$ saturation).

Fluorescein-labeling. HGG and rabbit antisera to HGG, MGG and guinea pig C'l were labe'ed with fluorescin isothiocyanate (Baltimore Biological Laboratory) by the method of Kawamura. ${ }^{21}$

Experimental procedure. The experimental mice were first injected in each hind footpad with $1 \mathrm{mg} \mathrm{HGG}$ in $0.05 \mathrm{ml}$ phosphate buffered saline. Four weeks later, these mice were boosted in the same manner as in the first injection and sacrificed at 0 and 1 hour and $1,3,6,8$ and 10 days after the second injection of antigen. They were killed by cutting throat and the blood was collected. Soon after the sacrifice, the popliteal lymph nodes were removed and processed for immunofluorescent and histologic examinations.

Immunofluorescence. Mouse popliteal lymph nodes fixed in cold $95 \%$ ethanol for 3 hours were embedded in paraffin and sectioned at $4 \mu$ in thickness. The sections were 
stained with fluorescein-labeled $\mathrm{HGG}$, or antisera to $\mathrm{HGG}$ or $\mathrm{MGG}$ for 30 minutes at $38^{\circ} \mathrm{C}$. For complement fixation test in tissues, some of the sections were incubated for 30 minutes at $38^{\circ} \mathrm{C}$ with fresh five-fold diluted guinea pig serum and then stained with fluoresceinated anti-guinea pig C'l antiserum. The stained sections were examined with a light mieroseope (Type S, Nippon Kogaku) equipped with a high pressure mercury bulb (AHL250, Perma Ray Industry), and photographed on Anscochrome 200 day light film. Specificity of staining reaction was determined by its blocking with non-conjugated HGG or globulin fractions of the appropriate antiserum. Control sections for complement fixation were incubated with guinea pig serum inactivated for 30 minutes at $56^{\circ} \mathrm{C}$ or with saline prior to the immunofluorescent staining.

Routine histology. The sections prepared as described above were stained with hematoxylin and eosin. The mean number of germinal centers in a single cross-section of one popliteal lymph node was calculated for each experimental group of mice.

\section{Results}

A characteristic curve of secondary serum antibody response is illustrated in Fig. 1. The serum antibody titer began to rise at day 3 and markedly increased until day 6 when the curve became flattened.

The mean number of germinal centers of all size per single cross-section of one popliteal lymph node is shown in Fig. 2. At 0 hour, the majority of popliteal nodes had only one or two germinal centers per section. Greater numbers were observed between days 3 and 10, with the peak number at days 6 to 8 .

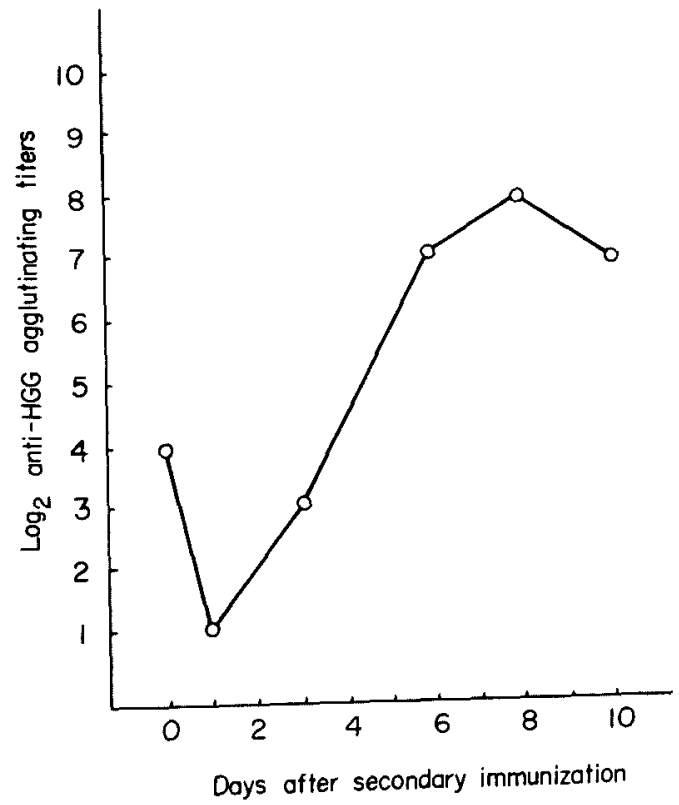

Fig. 1. Serum titers of anti-HGG agglutinating antibody in mice injected with a secondary dose of HGG. 


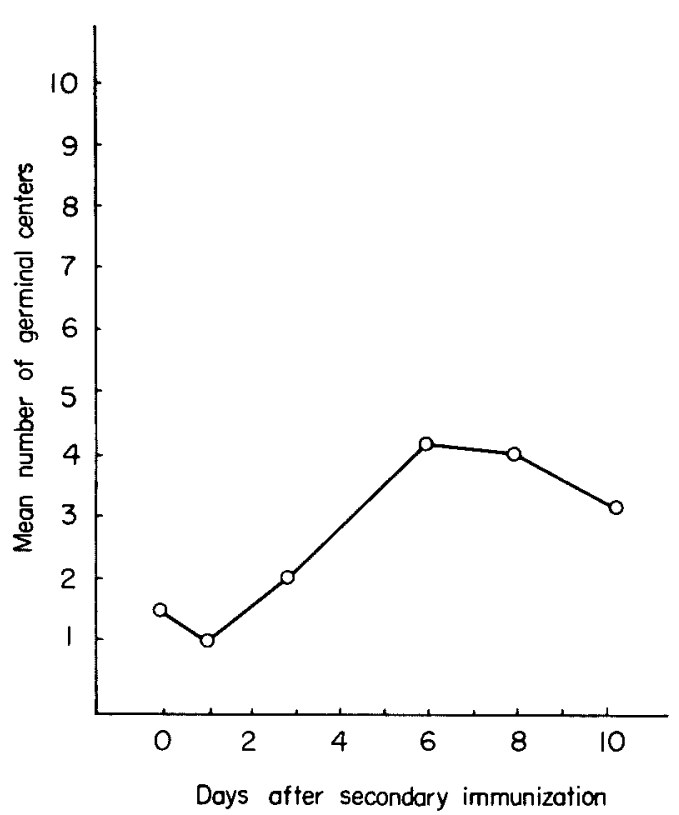

Fig. 2. Mean number of germinal centers in a single cross-section of one popliteal lymph node in mice injected with a secondary dose of HGG.

Localization of antigen. One hour after the booster injection of HGG, it was heavily localized in the germinal centers of popliteal lymph nodes (Fig. 3). The intense antigen localization in the germinal centers was also observed during the first three days (Figs. 4 and 5), followed by the diminution of immunofluorescence until day 10 when the antigen was hardly demonstrated in the germinal centers of popliteal nodes (Fig. 6). The distribution of antigen in the germinal centers was restricted to the interspace between germinal center cells, giving lacy or reticular framework of immunofluorescence. The germinal center cells were essentially free of the fluorescence.

In addition to the germinal centers, the positive fluorescence was also present over phagocytic cells lining medullary sinuses (Fig. 4). The apparent localization of antigen was sustained in the germinal centers up to day 8 , but the positive fluorescence in these phagocytic cells was only observed within 24 hours. At day 3 , when the germinal centers gave still brilliant fluoresence, the sinuses did not longer show any positive fluorescence.

Distribution of specific antibody-producing cells. Specific anti-HGG antibody producing cells were demonstrated in the sections of the popliteal lymph nodes by the fluorescent antigen staining. The majority of fluorescent cells were scattered through the medullary areas of popliteal lymph nodes (Fig. 7). The number of these cells was minimal during the first 24 hours after the secondary injection of $\mathrm{HGG}$ and moderate at day 3 , after which the fluorescent cells increased up to day 6 
(Fig. 7). These cells were present singly or in small clumps of several cells, and morphologically identified as plasmocytic elements of varying maturity ranging from large cells with narrow rim of fluorescent cytoplasm to smaller ones with more abundant area of fluorescent cytoplasm.

On the contrary, there were no fluorescent cells in the germinal centers through the period of observation, or if present, only a few number of plasma cells gave positive fluorescence (Fig. 8). In Fig. 9, a completely unstained germinal center demarcated from its medullary border by brightly fluorescent plasma cells is exemplified.

Localization of autologous $\gamma$-globulin. While the germinal centers did not show positive staining for specific anti-HGG antibody, autologous $\gamma$-globulin (MGG) was clearly demonstrated in the germinal centers of mouse popliteal lymph nodes (Figs. 10 to 12). The intensity of germinal center localization of MGG, which was slight at 0 hour, was increased up to day 6 and maintained till day 10 after the secondary injection of antigen (Figs. 10 to 12). The immunofluorescent pattern of positively stained germinal centers closely resembled that of distribution of antigen, which was located in the interspace between germinal center cells. The germinal center cells were essentially devoid of the specific fluorescence, although a number of plasma cells in the germinal centers contained $\gamma$-globulin (Fig. 11).

In the periphery of lymphoid follicles and in medullary cords, the majority of plasma cells also gave strong cytoplasmic fluorescence.

Fixation of heterologous complement. After the incubation of mouse popliteal lymph node sections with fresh guinea pig serum, it was possible to demonstrate positive fixation of guinea pig C' 1 in the germinal centers, which were stained in lacy appearance resembling the distribution of $H G G$ and $M G G$ (Fig. 13). The intensity of complement fixation in the germinal centers appeared to parallel that of $\gamma$-globulin localization. Control section incubated with saline or heat-inactivated guinea pig serum showed negative reaction.

\section{Discussion}

There is still controversy as to the role of germinal centers of lymphoid tissues in plasmocytopoiesis and/or lymphocytopoiesis despite numerous investigations in this field. In the previous ultrastructural study of human lymph node germinal centers, we concluded that germinal center cells might not be progenitors of plasma cells but they were capable of undergoing a lymphocytic differentiation. ${ }^{17}$ If one assumes the evolution of germinal center cells into plasma cells, then these cells may be capable of synthesizing specifis antibody or immunoglobulins. The first purpose of the present study was, therefore, to investigate the role of the germinal centers in the synthesis of specific antibody and immunoglobulin.

A survey of recent immunofluorescence studies dealing with the manner of immunoglobulin distribution in the germinal centers reveals two different groups of 
observation. Some investigators have described that immunoglobulins are located within the parenchymal cells of the germinal centers, ${ }^{11,15}$ while others have admitted that those are concentrated on the cytoplasmic processes of specialized reticular cells in the germinal centers, giving intercellular lacy framework of immunofluorescence. ${ }^{12-14,16}$ The results of the present study are not only in agreement with the latter observation but also indicate a close similarity of the distribution of $\gamma$-globulin to that of antigen in the germinal centers. Large parenchymal cells of the germinal centers were devoid of specific staining for $\gamma$-globulin as well as for specific antibody, whereas plasma cells distributed in the germinal centers and in perifollicular and medullary areas gave positive reaction. Furthermore, we could not confirm the fluorescence pattern of germinal centers indicating the transformation of germinal center cells into plasma cells as had been shown by Burtin and Buffe $^{15}$ and by Sordat et al. ${ }^{22}$ These findings may not favor the view that the germinal center is a source of plasma cells, but suggest that it is a site of lymphocyte formation.

It is well known that a specialized type of reticular cells is responsible for the localization and persistence of antigen in germinal centers during the immune reaction. ${ }^{23,24}$ The present study also showed the intercellular localization of antigen in the germinal centers, corresponding to the topographical distribution of specialized reticular cells. The similar germinal center localization of $\gamma$-globulin demonstrated in this study strongly suggests that the antigen was combined with specific antibody in the germinal centers. Specific fixation of heterologous complement in these parts further establishes the presence of an antigen-antibody complex mechanism. (We failed to detect specific antibody in the germinal centers by using fluorescent antigen staining because this staining was not adequate to demonstrate specific antibody, when it was combined with antigen injected). On the other hand, there is little question that antigen capture in germinal centers is greatly enhanced in the presence of specific antibody in actively $y^{8,9,25}$ as well as passively immunized animals. ${ }^{26,27}$ The mechanism involved in this phenomenon remains unexplained, but it may be assumed that the antigen complexed with specific cytophilic antibody is adsorbed to the cytoplasmic processes of specialized reticular cells for which specific cytophilic antibody has selective atfinity., 23,26 Several pertinent observations to support this idea have been made: 1) autologous $\gamma$-globulin reinjected into the foot-pads of rats appears to be strongly entrapped in the germinal centers of the draining lymph nodes;28 2) the germinal center localization of antigen is markedly depressed in immunologically tolerant ${ }^{29}$ or $x$ irradiated animals ${ }^{30}$ which neither possess nor produce detectable specific antibody; and 3) a primary dose of antigen-antibody complex leads to exaggerated follicular localization of antigen compared with that of antigen alone. ${ }^{27}$ We demonstrated further the existing antigen-antibody complex mechanism in the germinal centers, which also supports such an idea.

Although enhanced deposition of antigen in the germinal centers was observed during the first few days after the secondary immunization, this enhanced localiza- 
tion was followed by its elimination from the centers between days 6 and 10 . This process was observed in all germinal centers after the time when a large amount of $\gamma$-globulin was bound to the reticular network of germinal centers with a great deal of antigen. Since immunological complex adsorbed to cells often exert cytotoxic action against these cells especially in the presence of complement, ${ }^{31}$ it is possible that the loss of antigen-trapping capacity of the germinal centers results as a consequence of immunologic injury of specialized, antigen-retaining reticular cells caused by complement fixation. ${ }^{32}$ This receives a support from the observation of Gajl-Peczalska et al. ${ }^{16}$ that complement is actually localized in the germinal centers with a similar distribution to that of antigen and immunoglobulins.

The functional significance in the immune reaction of the germinal center localization of antigen remains a matter of speculation. Nossal and his coworkers presumed that lymphocytes undergoing blast transformation through the contact with antigen trapped in lymphoid follicles give rise to developing germinal centers at these sites. ${ }^{6}, 23$ On the basis of the present experiments, we could speculate further that a certain regulatory mechanism operates to control the development of germinal centers. During the secondary response, enhanced antigen deposition in the germinal centers would facilitate its contact with immunocompetent cells with higher affinity for antigen ${ }^{\mathbf{3 3 4} 3}$ and promote the growth of the germinal centers. However, this contact would be soon prevented by specific antibody produced, which competes with immunocompetent cells for antigen. When in the germinal centers, almost all antigen is combined with antibody, it is eliminated from the centers probably in conjunction with the loss of antigen-trapping capacity of specialized reticular cells, and consequently the germinal centers would become able to respond to renewed antigenic stimulation. Thus, the growth and development of germinal centers are considered to be regulated by a specific feedback mechanism mediated by antibody. The observation of Sahiar and Schwartz, ${ }^{\mathbf{5} 5}$ who showed the suppression of germinal center formation by the infusion of specific IgG antibodies, supports the possibility of our speculation.

\section{References}

1) Congdon, C.C. \& Makinodan, T. Splenic white pulp alteration after antigen injection. Relation to time of serum antibody production. Amer. J. Path., 1961, 39, 697-709.

2) Langevoort, H.L. The histophysiology of the antibody response I. Histogenesis of the plasma cell reaction in rabbit spleen. Lab. Invest., 1963, 12, 106-118.

3) Cottier, H., Odartchenko, N., Keiser, G., Hess, M. \& Stoner, R.D. Incorporation of tritiated nucleosides and amino acids into lymphoid and plasmocytoid cells during secondary response to tetanus toxoid in mice. Ann. New York Acad. Sci., 1964, $113,612-626$.

4) Movat, H.Z. \& Fernando, N.V.P. The fine structure of the lymphoid tissue during antibody formation. Exp. mol. Path., 1965, 4, 155-188.

5) Kaplan, M.H., Coons, A.H. \& Deane, H.W. Localization of antigen in tissue cells. III. Cellular distribution of pneumococcal polysaccharides types II and III in the mouse. J. exp. Med., 1950, 91, 15-30. 
6) Nossal, G.J.V., Ada, G.L. \& Austin, C.M. Antigens in immunity. IV. Cellular localization of ${ }^{125} \mathrm{I}$ - and ${ }^{131} \mathrm{I}$-labelled flagella in lymph nodes. Austr. J. exp. Biol. Med. Sci., 1964, 42, 311-330.

7) Sweet, L.C., Abrams, G.D. \& Johnson, A.G. The fate of radioactive bovine $\gamma$ globulin during the primary antibody response in the mouse. J. Immunol., 1965, 94, $105-110$.

8) McDevitt, H.O., Askonas, B.A., Humphrey, J.H., Schechter, I. \& Sela, M. The localization of antigen in relation to specific antibody-producing cells. I. Use of a synthetic polypeptide ((T,G)-A-L) labelled with iodine-125. Immunology, 1966, 11, 337-351.

9) Hanna, M.G., Jr., Francis, M.W. \& Peters, L.C. Localization of ${ }^{25}$ I-labelled antigen in germinal centers of mouse spleen. Effects of competitive injection of specific or non-cross-reaeting antigen. Immunology, 1968, 15, 75-91.

10) Ortega, L.G. \& Mellors, R.C. Cellular sites of formation of gamma globulin. $J$. exp. Med., 1957, 106, 627-640.

11) Mellors, R.C. \& Korngold, L. The cellular origin of human immunoglobulins $\left(\gamma_{2}\right.$, $\gamma 1 \mathrm{M}, \gamma 1 \mathrm{~A}) . \quad J . \exp$. Med., 1963, 118, 387-396.

12) Pernis, B. \& Chiappino, G. Identification in human lymphoid tissues of cells that produce group 1 or 2 gamma-globulins. Immunology, 1964, 7, 500-506.

13) Maruyama, K. \& Masuda, T. Electron microscopic observation on the germinal center of the lymph node in guinea pigs sensitized with sheep erythrocytes. Ann. Rep. Virus Res. Kyoto Univ., 1964, 7, 149-151.

14) Balfour, B.M. \& Humphrey, J.H. Localization of $\gamma$-globulin and labelled antigen in germinal centers in relation to the immune response. Germinal Centers in Immune Responses, edited by H. Cottier, N. Odartchenko, R. Schindler and C.C. Congdon, Springer, Berlin-Heidelberg-New York, 1967, pp. 80-85.

15) Burtin, P. \& Buffe, D. Synthesis of human immunoglobulins in germinal centers of lymphoid organs. J. Immunol., 1967, 98, 536-542.

16) Gajl-Peczalska, K.J., Fish, A.J., Meuwissen, H.J., Frommel, D. \& Good, R.A. Localization of immunological complexes fixing $\beta$ lc (C3) in germinal centers of lymph nodes. $J$. exp. Med., 1969, 130, 1367-1393.

17) Mori, M., Ishii, Y. \& Onoe, T. Studies on the germinal center. I. Ultrastructural study of germinal centers in human lymph nodes with correspondence to the zonal differentiation. J. Reticulo. Soc., 1969, 6, 140-157.

18) Sober, H.A. \& Peterson, E.A. Protein chromatography on ion exchange cellulose. Fed. Proc., 1958, 17, 1116-1126.

19) Nelson, R.A., Jensen, J., Gilgi, I. \& Tamura, N. Methods for the separation, purification and measurement of nine components of hemolytic complement in guinea-pig serum. Immunochemistry, 1966, 3, 111-135.

20) Daniel, T.M., Weyand, J.G.M., Jr. \& Stavitsky, A.B. Micromethods for the study of proteins and antibodies. IV. Factors involved in the preparation and use of a stable preparation of formalinized, tannic acid-treated, protein-sensitized erythrocytes for detection of antigen and antibody. J. Immunol., 1963, 90, 741-750.

21) Kawamura, A., Jr. Fluorescent antibody technique. Tanpakushitsu Kakusan Koso (Jap.), 1966, 11, 1621-1634.

22) Sordat, B., Moser, R., Gerber, H. \& Cottier, H. Differentiation pathway within germinal centers of human tonsils. Lymphatic Tissue and Germinal Centers in Immune Response, edited by L. Fiore-Fonati and M.G. Hanna, Jr., Plenum Press, New York, 1969, pp. 73-82.

23) Nossal, G.J.V., Abott, A., Mitchell, J. \& Lummus, Z. Antigens in immunity. XV. Ultrastructural features of antigen capture in primary and secondary lymphoid follicles. J. exp. Med., 1968, 127, 277-290.

24) Szakal, A.K. \& Hanna, M.G., Jr. The ultrastructure of antigen localization and viruslike particles in mouse spleen germinal centers. Exp. mol. Path., 1968, 8, 75-89.

25) Cohen, S., Vassalli, P., McCluskey, R.T. \& Benacerraf, B. Distribution of antigen in 
guinea pig lymph nodes during the primary and secondary immune response. Fed. Proc., 1965, 24, 696.

26) Nossal, G.J.V., Ada, G.L., Austin, C.M. \& Pye, J. Antigens in immunity. VIII. Localization of ${ }^{125} \mathrm{I}$-labelled antigens in the secondary response. Immunology, $1965,9,349-357$.

27) Ada, G.L. \& Lang, P.G. Antigen in tissues. II. State of antigen in lymph node of rats given isotopically-labelled flagellin, haemocyanin or serum albumin. Immunology, 1966, 10, 431-443.

28) Ada, G.L., Nossal, G.J.V. \& Austin, C.M. Antigens in immunity. V. The ability of cells in lymphoid follicles to recognize foreignness. Aust. J. exp. Biol. Med. Sci., $1964,42,331-346$.

29) Humphrey, J.H. \& Frank, M.M. The localization of non-microbial antigens in the draining lymph nodes of tolerant, normal and primed rabbits. Immunology, 1967, 13, 87-100.

30) Williams, G.M. Antigen localization in lymphopenic states. II. Further studies on whole body X-irradiation. Immunology, 1966, 11, 475-488.

31) Nelson, R.A., Jr. The role of complement in immune phenomena. The Inflammatory Process, edited by B.W. Zweifach, L. Grant and R.T. McCluskey, Academic Press, New York-London, 1965, pp. 819-872.

32) Hanna, M.G., Jr. \& Szakal, A.K. Localization of ${ }^{125}$ I-labeled antigen in germinal centers of mouse spleen. Histologic and ultrastructural autoradiographic studies of the secondary immune reaction. J. Immunol., 1968, 101, 949-962.

33) Bosman, C., Feldman, J.D. \& Pick, E. Heterogeneity of antibody-forming cells. An electron microscopic analysis. $J$. exp. Med., 1969, 129, $1029-1044$.

34) Sulitzeanu, D. \& Naor, D. The affinity of radioiodinated BSA for lymphoid cells. II. Binding of ${ }^{125} \mathrm{I}$-BSA to lymphoid cells of normal mice. Int. Arch. Allergy, 1969, $35,564-578$.

35) Sahiar, K. \& Schwartz, R.S. The immunoglobulin sequence. II. Histological effects of the suppression of $\gamma \mathrm{M}$ and $\gamma \mathrm{G}$ antibody synthesis. Int. Arch. Allergy, 1966, 29, $52-68$. 
Fig. 3. Distribution of antigen (HGG) in a germinal center of mouse popliteal lymph node 1 hour after secondary injection of HGG. $\times 300$.

Fig. 4. Localization of $\mathrm{HGG}$ in a germinal center and medullary sinuses of mouse popliteal node 1 day after secondary injection of HGG. $\times 150$.

Fig. 5. Intense localization of HGG in a germinal center of mouse popliteal node 3 days after secondary injection of HGG. $\times 170$.

Fig. 6. Localization of $\mathrm{HGG}$ in a germinal center of mouse popliteal node 6 days after secondary injection of $\mathrm{HGG}$. Note the reduction of immunofluorescence compared with that in Fig. $5 . \times 160$. 


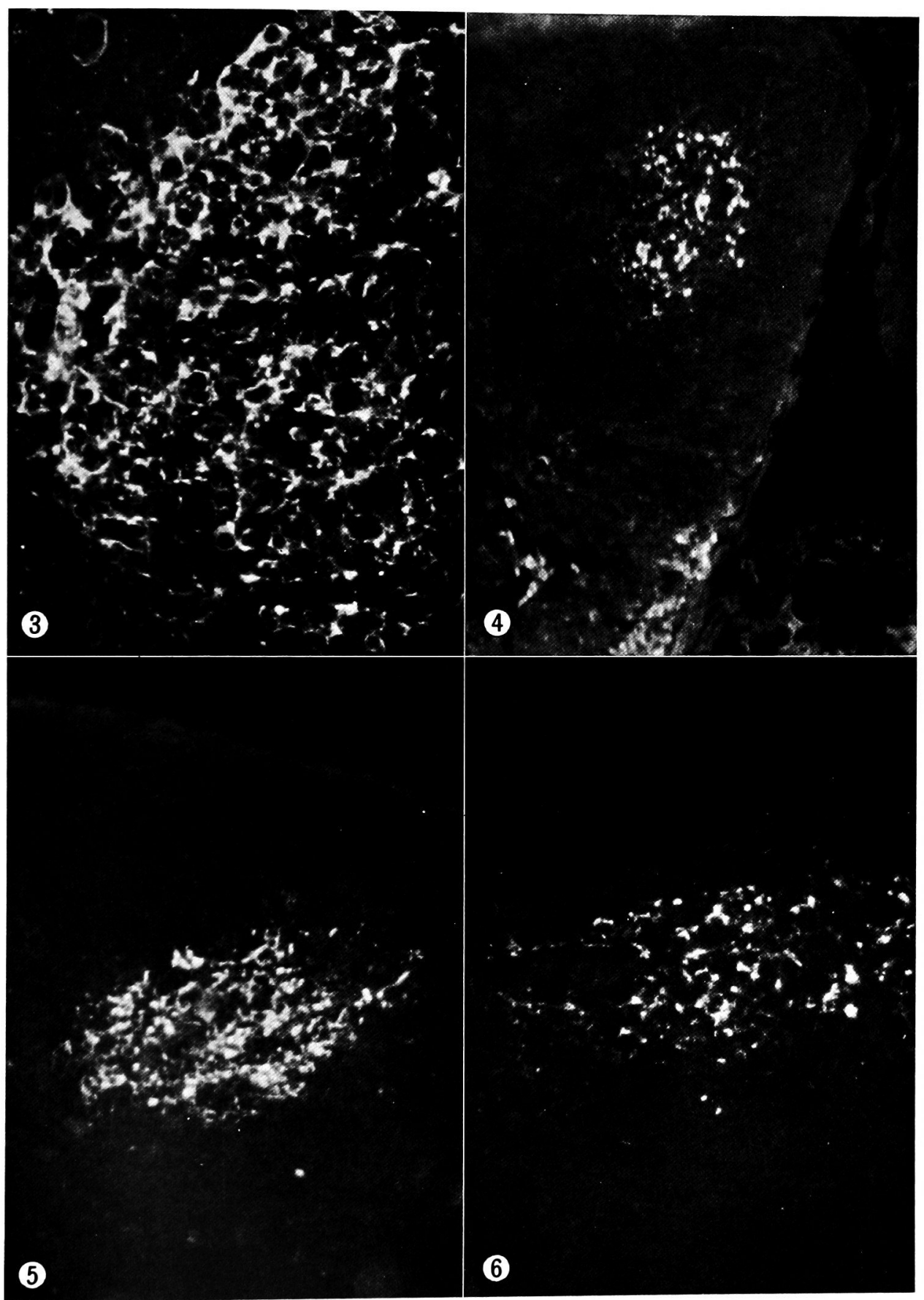


Fig. 7. Medullary area of mouse popliteal lymph node. Numerous plasma cells show specific staining for anti-HGG antibody (6 days after secondary stimulation with HGG). $\times 320$.

Fig. 8 A germinal center surrounded by fluorescent anti-HGG antibody-forming plasma cells. Germinal center cells are essentially devoid of specific staining for anti-HGG antibody except that a few number of plasma cells in the periphery of the germinal center show positive reactions ( 3 days after secondary stimulation with HGG). $\times 180$.

Fig. 9. Completely unstained germinal center demarcated from its medullary border by fluorescent plasma cells, stained with fluorescein-labeled HGG 6 days after secondary stimulation with HGG). $\times 150$. 
Germinal Center of Lymph Node in Secondary Immune Response

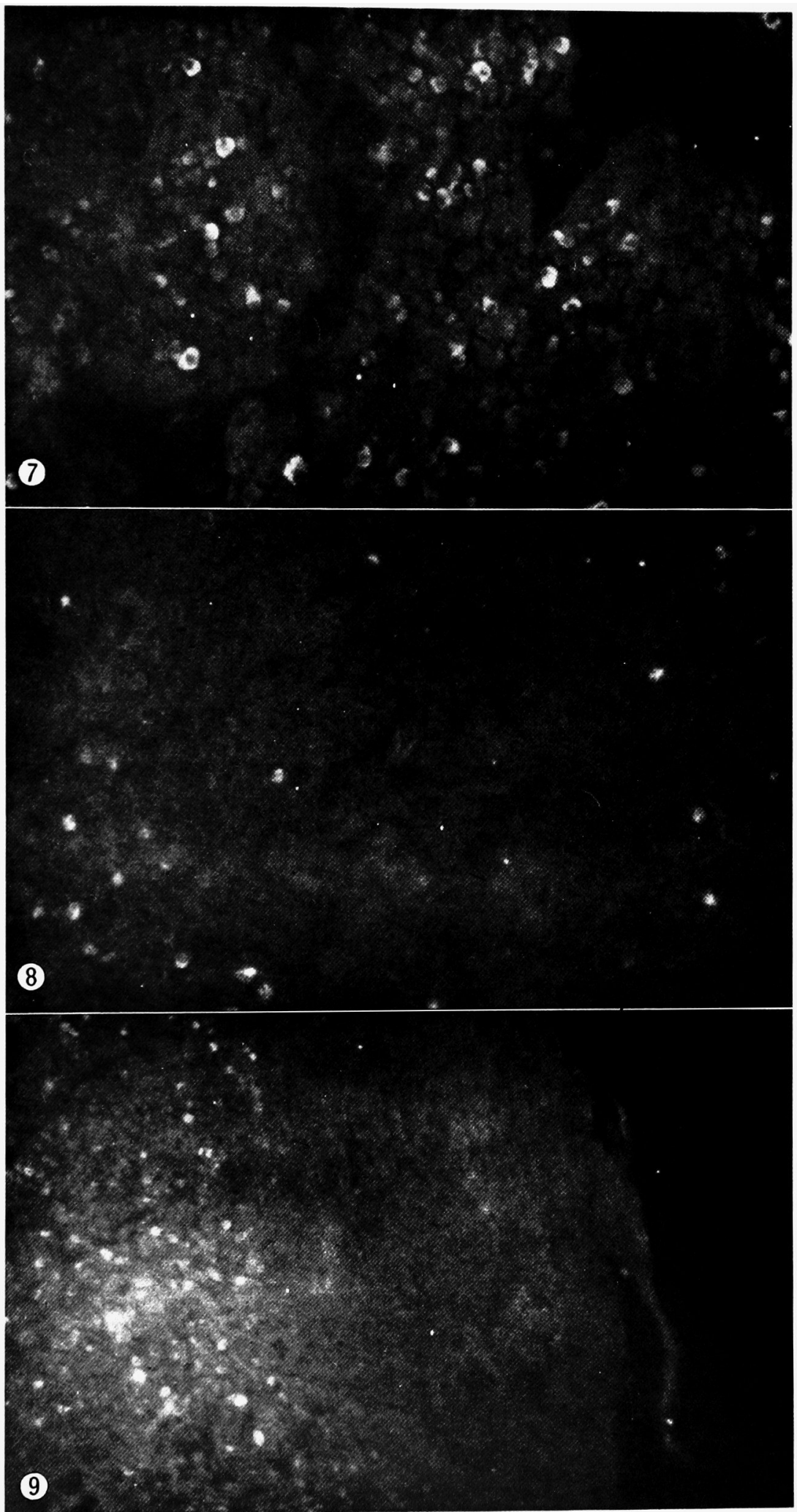


Fig. 10. Localization of autologous $\gamma$-globulin in a germinal center of mouse popliteal lymph node 1 day after secondary injection of HGG. $\times 280$.

Fig. 11. Distribution of autologous $\gamma$-globulin in a germinal center 3 days after secondary distribution similar to that of antigen (HGG). A few number of plasma cells present in the germinal center are also stained for $\gamma$-globulin. $\times 500$.

Fig. 12. Localization of autologous $\gamma$-globulin in a germinal center 6 days after secondary injection of $\mathrm{HGG}$, giving reticular network of immunofluorescence. $\times 250$.

Fig. 13. Fixation and distribution of guinea pig C'l in a germinal center of mouse popliteal lymph node 6 days after secondary injection of HGG. $\times 120$. 


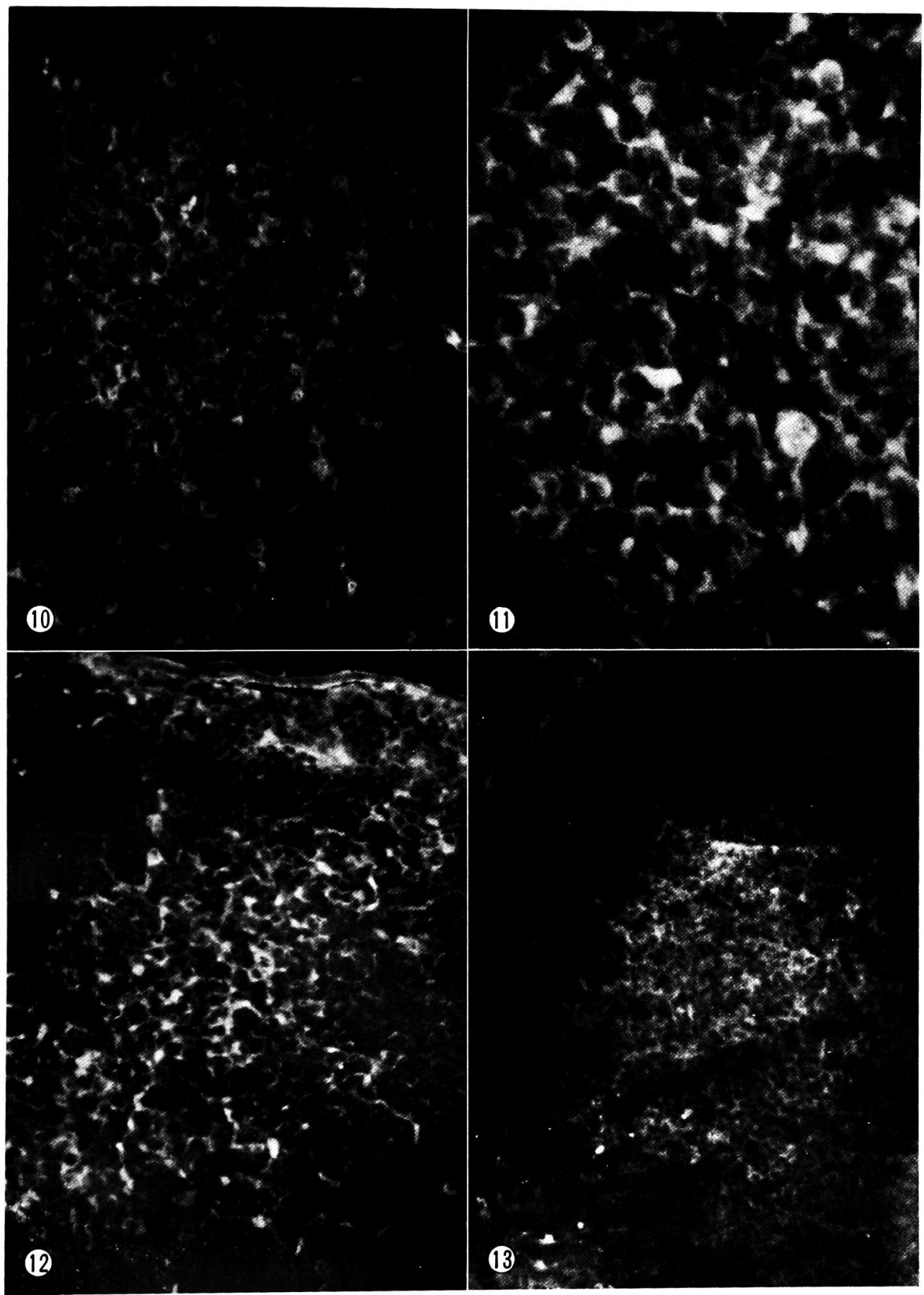

